\title{
Analgésie au long cours par transplantation subarachnoïdienne de cellules chromaffines
}

L'originalité extrême qui, pour un laboratoire de recherche, consiste à poursuivre seul pendant de longues années une voie de recherche est rare. Jacqueline Sagen et son équipe de l'University of Illinois à Chicago (USA) ont eu ce courage et en sont aujourd'hui récompensés. Au milieu des années 1980, les neurobiologistes et neurochirurgiens se sont enflammés pour les possibilités thérapeutiques nouvelles que semblaient offrir, pour la maladie de Parkinson, les transplantations intracérébrales de cellules chromaffines issues de la glande médullosurrénale. Fondées sur l'existence d'une synthèse massive de catécholamines dans ces cellules, les expériences chirurgicales se multiplièrent alors avec un succès finalement très mitigé et de graves problèmes postopératoires qui conduisirent rapidement à leur interruption. Jacqueline Sagen, alors jeune pharmacologue ayant surtout une expérience des problèmes d'analgésie expérimentale, eut l'idée d'utiliser les cellules chromaffines dans une tout autre optique. Outre des catécholamines, les cellules médullo-surrénaliennes produisent, en effet, d'importantes quantités de Met-enképhaline [1], un des neuropeptides inclus dans la famille des opiacés. Qui dit opiacés dit analgésie, et J. Sagen, associée à un neuroanatomiste, Georges Pappas, s'est lancée dans un programme de recherche visant à tester expérimentalement l'ensemble des paramètres à respecter pour utiliser ces cellules comme vecteur d'analgésie à long terme. Elle vient de présenter, lors du dernier congrès de la Society For Neuroscience à La NouvelleOrléans, les premiers résultats - positifs - d'essais cliniques directement issus de leur travail expérimental.

Sur la base d'expériences anciennes qui avaient démontré que l'injection intra- thécale d'enképhalines - dans l'espace subarachnoïdien qui sépare la moelle épinière de la dure-mère - induisait une analgésie importante [2], l'équipe de Chicago a réalisé des implantations subarachnoïdiennes de cellules chromaffines chez le rat. La technique expérimentale - qui est fondamentalement la même aujourd'hui dans les applications cliniques - consiste à couper longitudinalement la glande surrénale prélevée sur un donneur de façon à visualiser la medulla puis à séparer celle-ci du cortex par simple grattage. La medulla une fois isolée est découpée en morceaux d'à peu près $0,5 \mathrm{~mm}^{3}$. Ces morceaux sont pompés dans une aiguille dont la pointe est introduite dans l'espace sousarachnoïdien au niveau de la partie haute de l'élargissement médullaire lombaire, comme dans le cadre d'une ponction lombaire. Les morceaux de surrénale sont alors poussés à la surface de la moelle.

J. Sagen et son équipe ont démontré que les cellules chromaffines ainsi transplantées survivent et conservent une morphologie de type endocrinien [3]. Ces cellules sont fonctionnelles, puisqu'elles synthétisent et sécrètent d'importantes quantités de Metenképhaline dont les taux augmentent considérablement dans le liquide céphalo-rachidien des receveurs [4]. Surtout, la présence des cellules médullo-surrénaliennes modifie profondément la sensibilité des animaux à des stimuli nociceptifs ou à une stimulation douloureuse chronique. Les tests les plus généralement utilisés sont ceux qui étudient les réponses des animaux à des stimuli potentiellement dommageables pour les tissus sur lesquels ils sont appliqués (nociceptifs). On calcule généralement le temps pendant lequel le stimulus peut être appliqué avant que l'animal ne présente une réaction stéréotypée de défense ou d'évitement (figure 1). Il s'agit d'une évaluation de ce que l'on appelle, par référence aux sensations humaines, la douleur aiguë. Dans ces conditions, une transplantation subarachnoïdienne de cellules chromaffines ne modifiait pas, seule, les seuils de réponse. J. Sagen eut alors l'idée de sensibiliser le système en stimulant la libération du neuropeptide par une action sur les récepteurs nicotiniques que portent [5] les cellules chromaffines. La figure 1 montre que, dans cette situation de stimulation pharmacologique, tous les tests de nociception étaient modifiés. Cet effet antalgique de la greffe était inversé par l'injection systémique de naloxone, démontrant clairement l'implication des récepteurs opiacés.

La douleur aiguë n'est, toutefois, pas le problème majeur auxquels sont confrontés les cliniciens. Chez les malades douloureux chroniques, notamment chez les cancéreux en phase terminale, les systèmes physiologiques mis en jeu sont apparemment sensiblement différents de ceux impliqués dans la transmission des informations liées à la douleur aiguë. Depuis quelques années, on possède quelques modèles expérimentaux de douleur chronique chez lesquels on peut mieux apprécier les effets de traitements antalgiques au long cours sur le comportement de l'animal. Le mieux caractérisé de ces modèles est celui dit du " rat arthritique " [6] chez lequel un.syndrome polyarthritique est induit par injection souscutanée de Mycobacterium butyrion. C'est en étudiant ce modèle que l'équipe de Chicago a démontré effectivement l'applicabilité de son système à la douleur chronique humaine. En effet, alors que dans la douleur aiguë l'activité antalgique des cellules chromaffines 


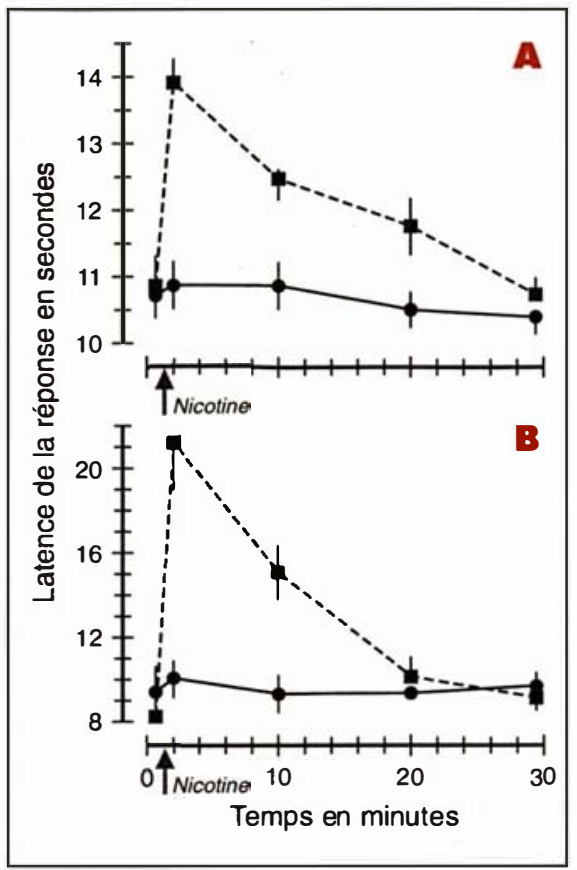

Figure 1. Délai de retrait de la patte (A) et de léchage des pattes (B) lors de I'application, respectivement, d'un pincement et d'une stimulation thermique nociceptive. Les résultats ont été obtenus après une injection souscutanée de nicotine $(0,1 \mathrm{mg} / \mathrm{kg})$ une minute après le début du test. En trait plein, animaux témoins; en pointillé, animaux greffés.

semblait liée à une stimulation pharmacologique, chez le rat arthritique existe un effet spontané du greffon [7]. Comme on le voit dans la figure 2, le rat arthritique est caractérisé par une rupture dans la pente de la prise de poids par rapport au rat sain. Cette perte de poids est liée à l'intensité du syndrome de douleur chronique et en est donc un reflet très utile. La greffe subarachnoïdienne de cellules médullosurrénaliennes a un effet tout à fait significatif sur ce paramètre en limitant notamment la perte de poids initiale. A La Nouvelle-Orléans, Jacqueline Sagen a donc présenté les résultats de la dernière étape de ses travaux [8] en montrant les effets antalgiques obtenus chez cinq patients cancéreux au stade terminal ayant reçu une implantation subarachnoïdienne de tissu médullo- surrénalien (obtenu dans une banque d'organes). Il est à noter que ces transplantations se sont accompagnées d'un protocole d'immunosuppression particulièrement léger puisque la ciclosporine A (10 mg/kg/jour) a été interrompue systématiquement au bout de quinze jours. Sur ces cinq patients, quatre ont présenté un effet spectaculaire se traduisant par l'arrêt de toute médication antalgique et une qualité de vie préservée jusqu'à leur décès, plusieurs mois après la greffe. Les concentrations de Met-enképhaline étaient très élevées dans le liquide céphalorachidien de tous ces patients. Les cellules médullo-surrénaliennes construisent donc le long de la moelle épinière une mini-pompe biologique capable de libérer au long cours des substances antalgiques - probablement opiacées - dans le liquide céphalo-rachidien de patients douloureux chroniques.

Malgré ce premier succès, une large diffusion de cette technique pourrait être lourdement freinée par le faible approvisionnement possible en médullosurrénales de donneurs. La médullosurrénale est, en effet, un tissu hautement vascularisé qui s'altère très rapidement dans des conditions d'anoxie. Le prélèvement sur des patients en coma dépassé est de ce fait problématique. Quoique la glande surrénale soit souvent prélevée en même temps que le rein dans le cas de tumeurs rénales, on ne peut compter sur un approvisionnement important au travers d'un tel protocole. Les tout derniers travaux de Jacqueline Sagen [9] pourraient, toutefois, apporter une solution à ce problème. Associée au groupe de Pat Aebischer (Brown University, Providence, USA), qui développe depuis des années des polymères implantables, elle a confectionné des polymères capables d'emprisonner des cellules chromaffines. Ces polymères sont ainsi faits que des petites molécules - comme les neuropeptides - peuvent diffuser dans le tissu alors que les cellules implantées ne peuvent entrer en con-

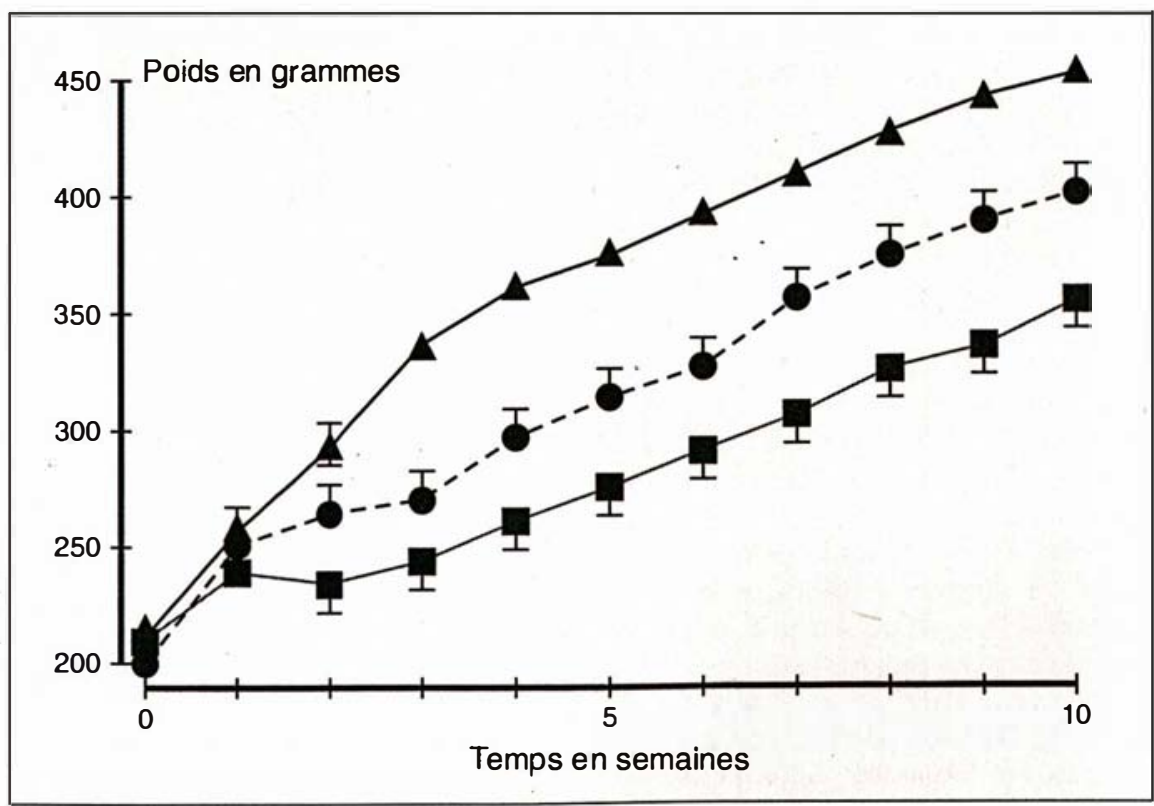

Figure 2. Pentes de la prise de poids au cours du temps chez des rats témoins (triangles noirs) et chez des rats rendus arthritiques au temps 0 par injection de Mycobacterium butyricum. Les carrés noirs montrent les résultats obtenus chez des animaux non greffés, les ronds noirs ceux obtenus chez des rats porteurs d'une transplantation chromaffine subarachnoïdienne. 
tact avec celles de l'hôte, évitant ainsi un rejet éventuel. Les xénogreffes sont aujourd'hui possibles sans immunosuppression entre le bœuf et le rat... à quand des greffes de médullosurrénales bovines chez l'homme?

Marc Peschanski

\section{RÉFÉRENCES}

1. Livett B, Dean DM, Whelan LG, et al. Co-relcase of enkephalin and catecholamines from cultured adrenal chromaffin cells. Nature $1981 ; 289$ : 317-9.

2. Inturrisi CE, Umans JG, Wolff $\mathrm{D}$, et al. Analgesic activity of the naturally occurring hectapeptide (Met)enkephalin-Arg6-Phe ${ }^{7}$. Proc Natl Acad Sci USA 1980 ; 77 : 5512-4.

3. Sagen J, Pappas GD, Morphological and functional corrclates of chromaffin cell transplants in CNS pain modulatory regions. Ann NY Acad Sci 1987 ; 495 : 306-33.

4. Sagen J, Kemmler J. Increased levels of Met-enkephalin-like immunoreactivity in the spinal cord CSF of rats with adrenal medullary transplants. Brain Res $1989 ; 502: 1-10$.

5. Sagen J. Pappas GD, Pollard HB. Analgesia induced by isolated bovine chromaffin cells implanted in rat spinal cord. Proc Natl Acad Sci USA 1986 ; 83 : 7522-6.

6. Calvino B, Crepon-Bernard MO, Le Bars D. Parallel clinical and bchavioural studies of adjuvant-induced arthritis in the rat : possiblc relationship with "chronic pain ". Behav Brain Res 1987 ; 24 : 11-29.

7. Sagen J, Wang H, Pappas GD. Adrenal medullary implants in the rat spinal cord reducc nociception in a chronic pain model Pain $1990 ; 42$ : 69-79.

8. Sagen J, Winnie AP, Wang $\mathrm{H}$, et al. Pain reduction by adrenal medullary transplants in the spinal subarachnoid space of terminal cancer patients. Neurosci Abst 1991; $17: 235$

9. Wang H, Tresco PA, Aebischer P, Sagen $\mathrm{J}$. Pain reduction by transplants of polymer cncapsulated bovine chromaffin cells in the rat subarachnoid space. Neurosci Abst 1991; 17 : 235

\section{TIRÉS A PART}

M. Peschanski. 